\title{
Dynamic Vehicle and Rigid Road Interaction Analysis and Vibration Control of the Quarter Car Model Using Adaptive Neuro-Fuzzy Algorithm
}

\author{
${ }^{1}$ Mehmet Akif Koç \\ ${ }^{* 1}$ Faculty of Technology, Department of Mechatronics Engineering Sakarya Applied Sciences University, Turkey
}

\begin{abstract}
In this study 3-DOF quarter car model with the three bumps on the rigid road, the assumption has been modeled with the non-random irregularity. To reduce the excessive vibrations occurred on the vehicle body, an active suspension system with the linear actuator has been considered. Moreover, to control this actuator, an adaptive neuro-fuzzy algorithm is designed. The training and testing data of the ANFIS has been obtained from Proportional Integral Derivative (PID) control algorithm. After that the successful training process, a testing procedure has been applied to ANFIS for the measure of the adaptive neuro-fuzzy system with data that are not considered in the training process. Then, the performance of the ANFIS is compared by the PID algorithm and passive suspension system in terms of vehicle body vertical acceleration, vehicle body vertical displacement, and control force. The road model used in the study has been modeled according to non-random road profile mathematical formulation considering periodical and discrete road profile cases. In this formulation, one can easily determine the height, width, and number of the road defect with the series mathematical formulation. Consequently, with the results obtained from the presented study, it is proven that ANFIS is a very effective controlling algorithm to suppress vibration occurred on the vehicle body due to vehicle road interaction. Furthermore, the performance of the ANFIS has been tested with different parameters, for example, different number membership functions (MF), which used the fuzzification of the input parameters.
\end{abstract}

Key words: Active suspension, ANFIS, vibration control, quarter car model.

\section{Introduction}

The road and vehicle tire interaction is a critical issue in evaluating passenger comfort, driving safety, and vehicle mechanical competent service life because the forces transmitted to the vehicle negatively affect this parameter. Increasing the forces transmitted to vehicle components causes reducing vehicle components and pavement's practical lifetime. Moreover, the vibrations transmitted from the road affect passenger comfort due to the vertical disturbance movement and acceleration vehicle body. A vehicle tire, in normal working conditions, has to be in contact with the foundation. Due to vehicle mass, the vehicle tire starts to deflect, and this deflection is very considerable because of its flexibility [1]. The researchers reported that the linear mechanical laws couldn't explain this deflection under the normal load. For this reason, some non-linear tire models have been proposed by the researchers [2,3]. It is clear that the deflection amount of the tire at the contact point with the rigid road is related to the load implemented to tire axes vertically.

The road bumps in highway transportation have vital applications for traffic safety $[4,5]$. When a car moving o traffic conditions, this car can be various types of road irregularities. These

*Corresponding author: Address: Faculty of Technology, Department of Mechatronics Engineering Sakarya Applied Sciences University, 54187, Sakarya TURKEY. E-mail address: makoc@ subu.edu.tr, Phone: +902646160942 
irregularities can be random or built for any purpose with their location, depth, height, and number [6,7]. several active and passive systems of the vehicle, simulation computer tools have been developed by the researchers to cope with this irregularity. Suitable bumps corresponding to any speed have been designed, and results are compared with the experimental using the computer simulations tools data. When a vehicle moves on a bump, several parameters can be considered for determining dynamic vehicle responses. The most critical parameters that affect the vehicle dynamics are dimensions, geometry, and a number of the bumps and suspension dynamic characteristic properties of the vehicle. If the bumps are not correctly designed or dimensioned, the vehicle component can be damaged, and thus, this situation can affect vehicle safety [8,9]. Although the growing number of traffic elements and their apparent effect on vehicle dynamic responses, very few studies analyze their effect on road safety, passenger comfort, or deterioration of vehicles [10,11]. Moreover, it should be noted that vans, SUVs, or off-road vehicles with a high gravity center are especially sensitive to these bumps. Consequently, investigating the different types of bumps considering different vehicle speeds and different types of vehicles is a considerable research area in terms of analyzing bumps' effect upon stability, comfort, and safety [12].

The 3-DOFs quarter car vehicle model with the vehicle tire, vehicle suspension, and the vehicle body is studied. The vehicle considered moving on the rigid road in which three bumps included to surface. For the controlling vehicle body vibration, the PID and ANFIS control algorithm has been designed. The ANFIS training and testing data have been obtained from the PID control algorithm. Then, the performance of the ANFIS is compared with the PID algorithm. Consequently, it is proven that using the PID data for the ANFIS control algorithm is very effective in terms of the 3-DOFs quarter vehicle vibrations effect of the rigid road with the nonrandom bumps. The presentation of the study has been organized as follows: the mathematical formulation of the vehicle, road, the PID control algorithm, and ANFIS architecture is given in Section 2. Moreover, the analysis results of the study are presented in section 3.

\section{Mathematical modeling}

\subsection{Rigid road and vehicle mathematical modeling}

In this study, for the mathematical modeling of the vehicle and rigid road interaction, the physical model given in Figure 1 is considered. As shown in figure three DOFs quarter car model vehicle moving with constant speed $v$ on the rigid road with the non-random road irregularity considering three bumps is given. The non-random road irregularity given in Figure 1 can be considered periodical and discrete. According to the mathematical background, the non-random road irregularities can be stated as follows:

$r_{d}(x)=\frac{1}{2} \zeta(1-\cos 2 \pi x / \gamma)$

Here $\zeta$ represents the depth or height of the road defect, and $\gamma$ represents its width. Equation (1) is expressed as a series as in Eqs. (15a-b). 
$r_{d}(x)=\left\{\begin{array}{l}\frac{1}{2} \zeta\left(1-\cos \left(\frac{2 \pi(x-C)}{\gamma}\right)\right), \text { for } C \leq x \leq C+\gamma \\ 0 \quad \text { elsewhere }\end{array}\right.$

$C=B+k(A+\gamma), \quad k=0,1, \ldots, N_{i}$

Here $B$ is the distance between the defect on the bridge and the left end of the bridge, which is taken as the reference point, $L_{d}$ represents the distance between two consecutive road defects, and $N$ refers to the number of defects.

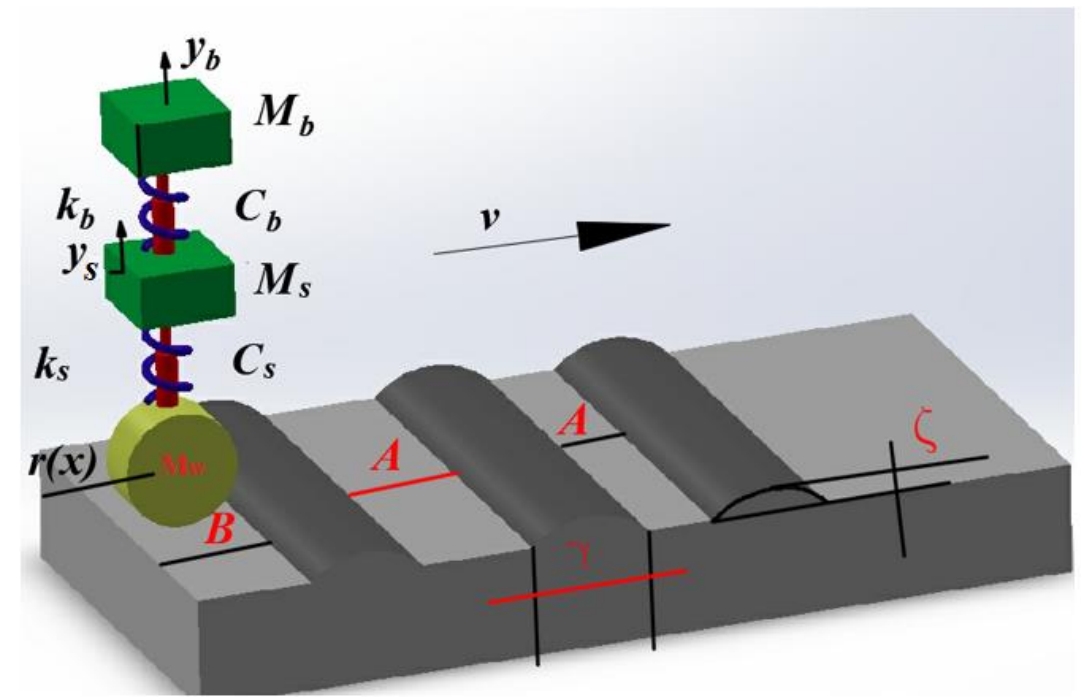

Figure 1. A 3-DOF quarter car model is moving on the rigid road with three bumps.

Moreover, for the investigation quarter car model in Figure 1, the parameters $k_{s}$ and $k_{b}$ represent suspension spring coefficient and driver seat spring coefficient, respectively. Furthermore, the parameter $C_{s}$ and $C_{b}$ represent suspension damping coefficient and driver seat damping value, respectively. The vehicle's tire, suspension, and body mass have been defined by parameters $M_{W}$, $M_{S}$, and $M_{b}$, respectively. The parameters $r(x), y_{s}$ and $y_{b}$ represent tire vertical displacement or non-random road profile, suspension vertical displacement and vehicle body vertical displacement. For the mathematical modeling following assumptions are considered:

- The vehicle's moving speed is considered constant.

- There are three bumps on the rigid road.

- Only one car moving on the road is taking into consideration.

For the quarter car model given in Figure 1, the kinetic and potential energy of the system can be written as follow respectively: 


$$
\begin{aligned}
& T=\frac{1}{2}\left(M_{w} \dot{r}(x)^{2}+M_{s} \dot{y}_{s}^{2}+M_{b} \dot{y}_{b}^{2}\right) \\
& V=\frac{1}{2}\left(k_{s}\left(y_{s}-r(x)\right)^{2}+k_{b}\left(y_{b}-y_{s}\right)^{2}\right)
\end{aligned}
$$

On the other hand, the damping function of the vehicle system can be obtained by as follow:

$$
D=\frac{1}{2}\left(C_{s}\left(\dot{y}_{s}-\dot{r}(x)\right)^{2}+C_{b}\left(\dot{y}_{b}-\dot{y}_{s}\right)^{2}\right)
$$

The Equation of motion of the quarter car vehicle model can be governed by the Lagrange equation $(L)$ given by Equation (7):

$$
\frac{\mathrm{d}}{\mathrm{d} t}\left(\frac{\partial L}{\partial \dot{p}_{k}(t)}\right)-\frac{\partial L}{\partial p_{k}(t)}+\frac{\partial D}{\partial \dot{p}_{k}(t)}=U_{k}, k=1,2, \ldots ., 3
$$

The state variables given in Equation (7) can be written as follows:

$$
\mathbf{p}=\left\{\begin{array}{lll}
r & y_{s} & y_{b}
\end{array}\right\}
$$

Using Equations (4-6) and the Lagrange equation given in Equation (7), the equations of motion of the vehicle system are described as follow:

$$
\begin{aligned}
& M_{w} \ddot{r}-k_{s}\left(y_{s}-r\right)-C_{s}\left(\dot{y}_{s}-\dot{r}\right)=0 \\
& M_{s} \ddot{y}_{s}+k_{s}\left(y_{s}-r\right)-k_{b}\left(y_{b}-y_{s}\right)+C_{s}\left(\dot{y}_{s}-\dot{r}\right)-C_{b}\left(\dot{y}_{b}-\dot{y}_{s}\right)=0 \\
& M_{b} \ddot{y}_{b}+k_{b}\left(y_{b}-y_{s}\right)-C_{b}\left(\dot{y}_{b}-\dot{y}_{s}\right)=0
\end{aligned}
$$

\subsection{PID modeling to obtain training data for ANFIS}

In this study, the training and testing data for ANFIS have been obtained from the PID algorithm. PID controller is one of the first control strategies. The control cycle is shown in Figure 2 $[13,14]$. A simple PID controller structure consists of $K_{p}, K_{d}, K_{i}$ with proportional, integral, and derivative gains, respectively. There are several different types of PID controllers. These are parallel and serial controllers, and Fig. 2b shows the parallel controller design. The parallel structured PID controller (from now on referred to as the PID controller) collects all error signals e (t) and then generates the input signal $u(t)$ after multiplying this signal by the PID gains $K_{p}, K_{d,}$ and $K_{i}[15]$. 
Figure 2 shows that conventional feedback PID control algorithm closed-loop schema. As shown in the figure PID control algorithm consists of one input (reference input for the control algorithm) and one output signal of the plant system. This output signal is detected by a sensor and then this signal is compared with the input reference signal using comparison element as shown in Figure 2. The error signal has been obtained by this comparison. Then, this error signal has been transferred to gain, integral and derivative elements for processing in these elements. Using these processed signals at the gain, integral and derivative element with the sum of them, the control output of the PID algorithm has been generated. This signal is transferred to the plant system (in this study, the plant system is a quarter car vehicle active suspension system) to control the output signal generated by the system. Then, according to this control force, the system's output has been obtained. The control output $U(t)$ for the quarter car vehicle body actuators has been obtained as shown in Equation (10), respectively using the error signal generated by the PID control algorithm between the reference signal and the output signal of the plant system.

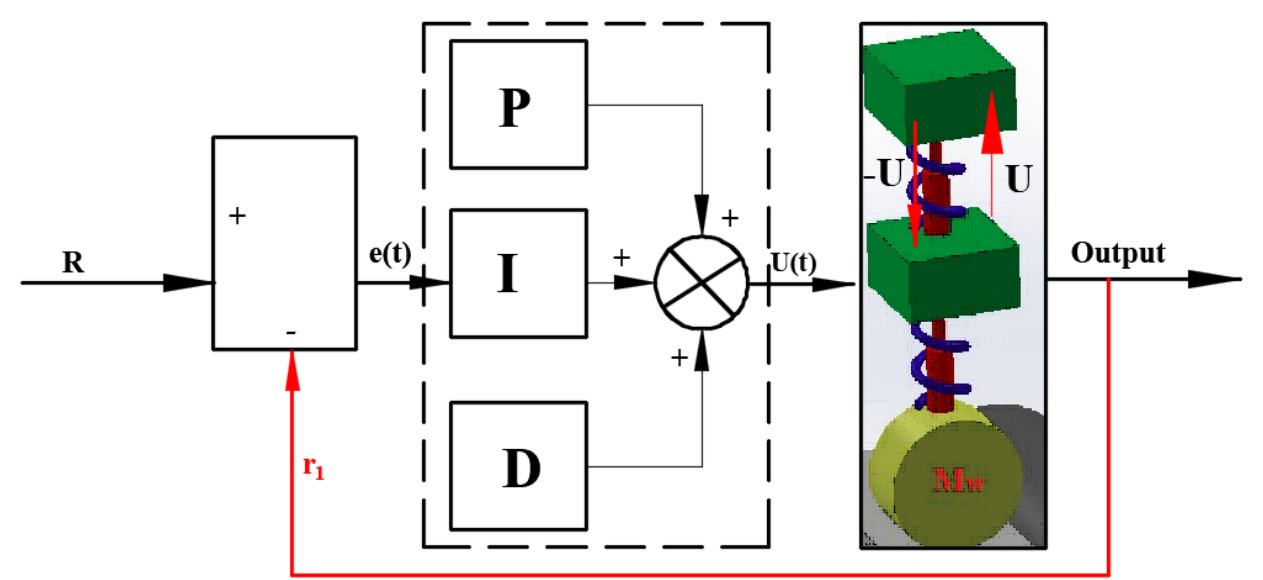

Figure 2. The PID control schema for the quarter car vehicle.

$U(t)=K_{p} e(t)+K_{i} \int_{0}^{t} e(t) d t+K_{d} \frac{d e(t)}{d t},(i=1,2),\left\{e=0-y_{b}\right\}$

On the other hand, the parameters $K_{p}, K_{i}$, and $K_{d}$ given in Equation (10) represent respectively proportional, integral, and derivative gains of the PID controller. . The control signal generated by sensors attached to the vehicle body has been transferred to the PID controller then a control signal produced by the controller using Eq. (10), then, this control signal. In this considering proportional, integral, and derivative gains $K_{p}=1984, K_{d}=25765$, and $K_{i}=2268$ respectively.

\subsection{Modelling of the ANFIS control algorithm}

ANFIS deals with the Adaptive Neuro-Fuzzy Inference System controller. The ANFIS is a Fuzzy logic control system integrated with Artificial Neural Network (ANN), whose membership functions (MF) and control rules are constructed adaptively using Neuro-adaptive learning methods. Generally, ANFIS is occurred by five-layer architecture, as shown in Figure 3. Each 
layer has a predefined specific function, and all of these layers are used for ANFIS model development. One level of this hybrid learning is called as epoch. The MATLAB toolbox "Neuro-Fuzzy Designer" is used to generate the ANFIS model

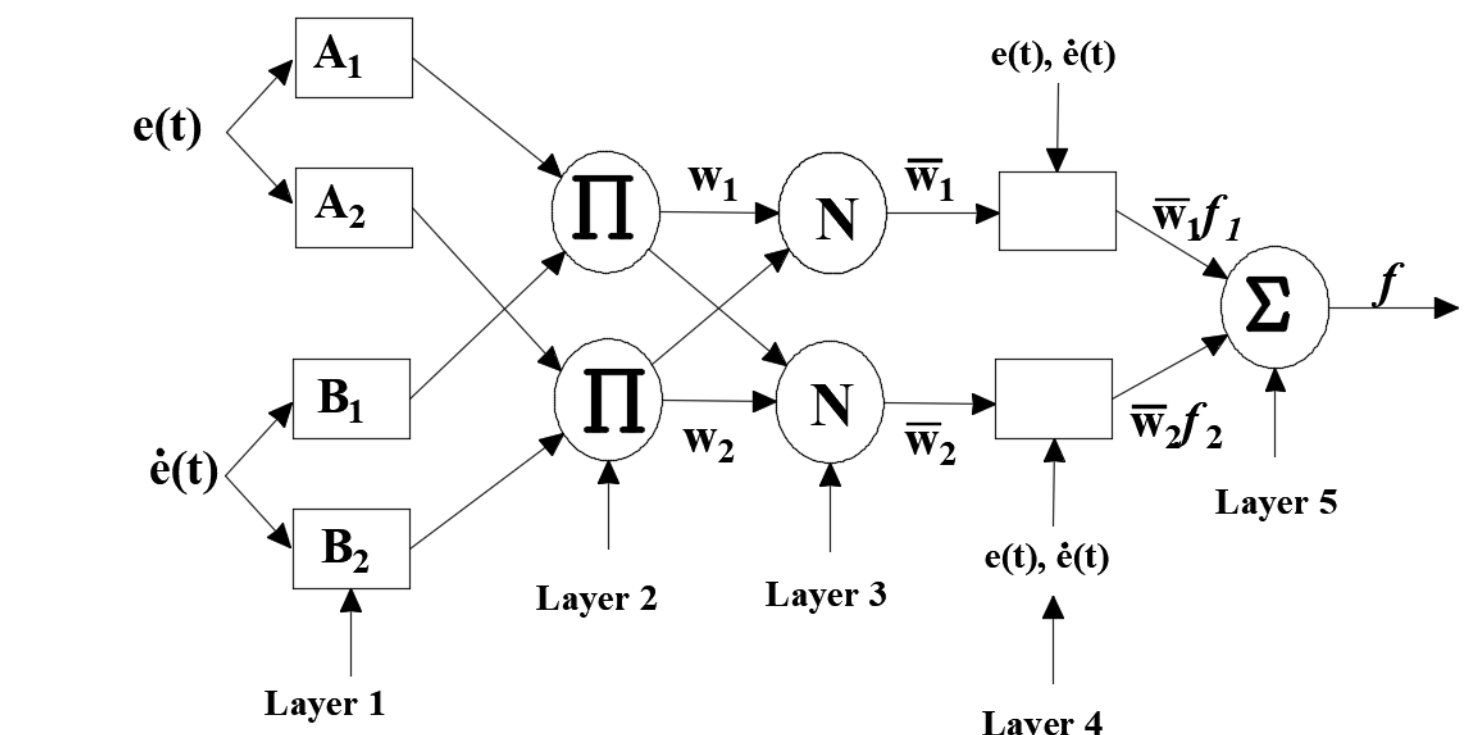

Z

Figure 3. The ANFIS architecture was used in this study.

Layer 1: This layer is comprised of the square node and adapts to the functional parameter at the input. Each node output is the degree of membership value that is given by the input of MF.

Layer 2: This layer is comprised of the circle node and determines the triggering strength of every rule by using the multiplying operator. The result at the output is obtained by multiplying the input signal and signal delivered to the next node.

Layer 3: This layer is comprised of the circle node that determines the triggering strength of rules to the sum of triggering strength of all the rules. This results in a normalized triggering strength.

Layer 4: This layer is comprised of the square node, which calculates the parameter function known as consequent parameters.

Layer 5: This layer node calculates the sum of signals at the input as output.

Let us assume that in a double-input $(x, y)$ parameter system, fuzzification of the parameter $x$ is represented by $n$ number of MFs, and fuzzification parameter of $y$ is represented by $m$ number of MFs. In this case, $N_{R}$ is the number of rules, $N_{R}=m \times n$ number of rules are written in Takaki- 
Sugeno type IF-THEN form to define the relation between ANFIS input parameters and output parameters.

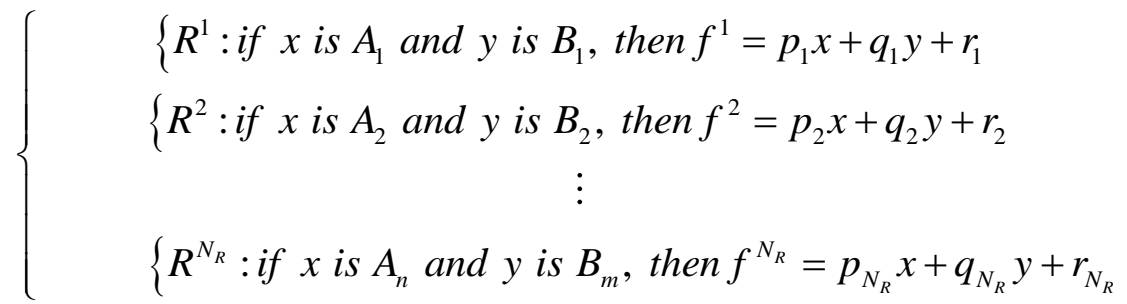

In Equation (11) $p_{i}, q_{i}$ and $r_{i}$ are consequent parameters which are determined during the training of ANFIS.

\section{Results}

For the numerical analysis, three different control models (passive control, PID control, and ANFIS control) have been investigated for the comparation of the control performance of the three-DOFs vehicle moving on the rigid road with the three bumps shown in Figure 1. The first model given in Figure 1 has a passive vibration system to control vibration due to interaction between moving three-DOFs vehicle and rigid road. This model doesn't contain any vibration control algorithm. The second one is the PID control algorithm to control vibration, which schematic diagram is given in Figure 2. The PID algorithm consists of one input and output parameter. The input parameter is the reference parameter represented by parameter $R$. The reference parameter has been defined by value zero. On the other hand, the output parameter of the PID algorithm is defined by the vertical displacement of the three-DOFs vehicle body, which is represented by parameter $\mathrm{y}_{b}(t)$. In this study, the PID algorithm has been used for the two different objectives. One of these objectives why PID algorithm is used in this study is obtaining training data far ANFIS algorithm which third control model used the presented study. The second objective of the PID algorithm used in the study is to compare the performance of the ANFIS algorithm with the different control algorithms. In this section, the ANFIS control algorithm, which is explained in the previous detail section, has been trained according to data obtained from the PID control system. For this, firstly, a PID control algorithm is performed for the suppression vibration due to interaction between three-DOFs vehicle suspension. The constant speed of the three-DOFs vehicle suspension $v=20 \mathrm{~m} / \mathrm{s}$. In this paper, optimal PID gain values for the specified plant model are obtained as $K_{p}=1984, K_{d}=25765$, and $K_{i}=2268$ for three-DOFs vehicle suspension. For the numerical analysis, the time step size is defined as $\Delta \mathrm{t}=0.001 \mathrm{~s}$.

For the numerically analysis, $M_{w}=10 \mathrm{~kg}, M s=100 \mathrm{~kg}, M_{b}=100 \mathrm{~kg}, C_{s}=2000 \mathrm{Ns} / \mathrm{m}^{2}, C_{b}=300$ $\mathrm{Ns} / \mathrm{m}^{2}, K_{s}=628 \mathrm{~N} / \mathrm{m}, K_{b}=500 \mathrm{~N} / \mathrm{m}$ are considered. The total length of the road is taken as $L=100$ $\mathrm{m}$. On the other hand, for the road parameters, the number of defects on the road $N=3$, the height of the defect $\zeta=0.01 \mathrm{~m}$, the width of the defect on the road $\gamma=0.5 \mathrm{~m}$, the parameter $A=5$, and $B=12.5 \mathrm{~m}$ are taken into consideration. For the ANFIS simulation, the number of membership 
functions (MF) to use fuzzification of the input parameters are considered MFs=5. ANFIS training process has been completed by 25000 training cycles with the medium capacity computer (i7 processor, 32 GB RAM). In the training set, a 50 number training pattern has been included. Finally, Figure 4 shows the training performance of ANFIS according to the actual value of the time-dependent actuator forces according to input values error signal and derivative of the error signal. As seen shown in Figure 4, it is seen that the actuator force very increased when the car is moving over the bumps. On the other hand, for the ANFIS training process, MSE and $\mathrm{R}^{2}$ values have been obtained as 1520 and \%85, respectively. Figure $4 \mathrm{~b}$ shows that ANFIS testing performance for 50 number patterns in the testing set with the MSE and R2 values are 1580 and \%82, respectively.
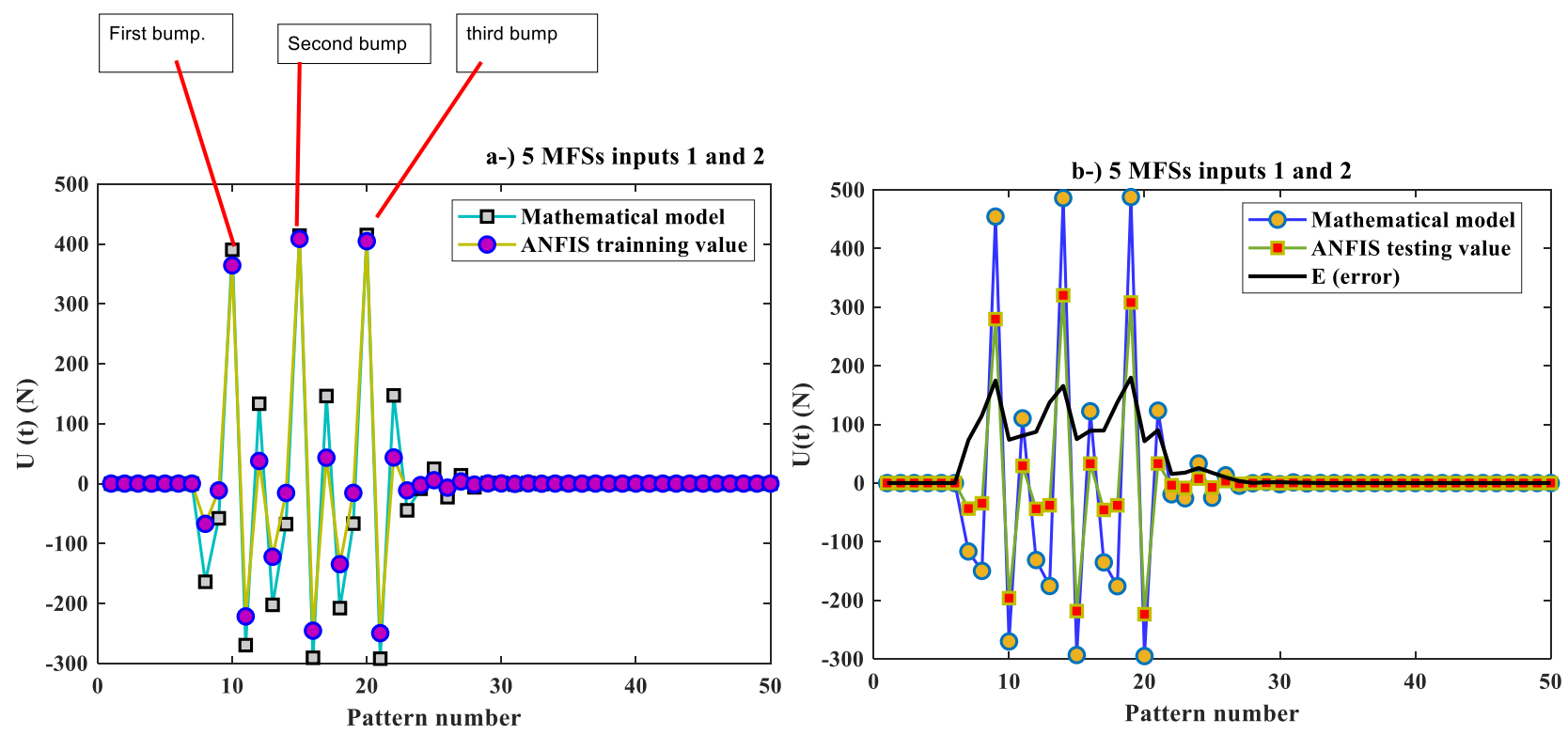

Figure 4. The ANFIS performance, a-) for the training, b-) for the testing.

Moreover, Figure 5a shows a comparison of the three different control algorithms presented in this study according to vehicle body vertical displacement of the 3-DOFs quarter car model given in Figure 1. As shown in the figure, in the passive suspension system, maximum vehicle body vertical displacement has been obtained as $7.54 \mathrm{~mm}$. it is seen that the ratio of $\% 87$ decreases the vertical displacement of the vehicle body. On the other hand, for the PID and ANFIS algorithms, this value is 1.32 and $1.74 \mathrm{~mm}$, respectively. According to this comparison, the performance of the PID is better than the ANFIS control algorithm. Figure 5b shows vehicle body vertical acceleration in three different control algorithms (passive suspension, PID control algorithm, and ANFIS control algorithm). As shown in the figure, maximum vehicle body vertical accelerations have been obtained as $0.23,0.38$, and $0.37 \mathrm{~m} / \mathrm{s} 2$, respectively. Moreover, Figure 5c shows control forces which are represented by ANFIS's output for the two-control algorithms (the PID control algorithm and the ANFIS control algorithm). According to the figure, the maximum control force of the PID algorithm is obtained as $519 \mathrm{~N}$. On the other hand, maximum control forces for the ANFIS control algorithm are obtained as $584 \mathrm{~N}$. 

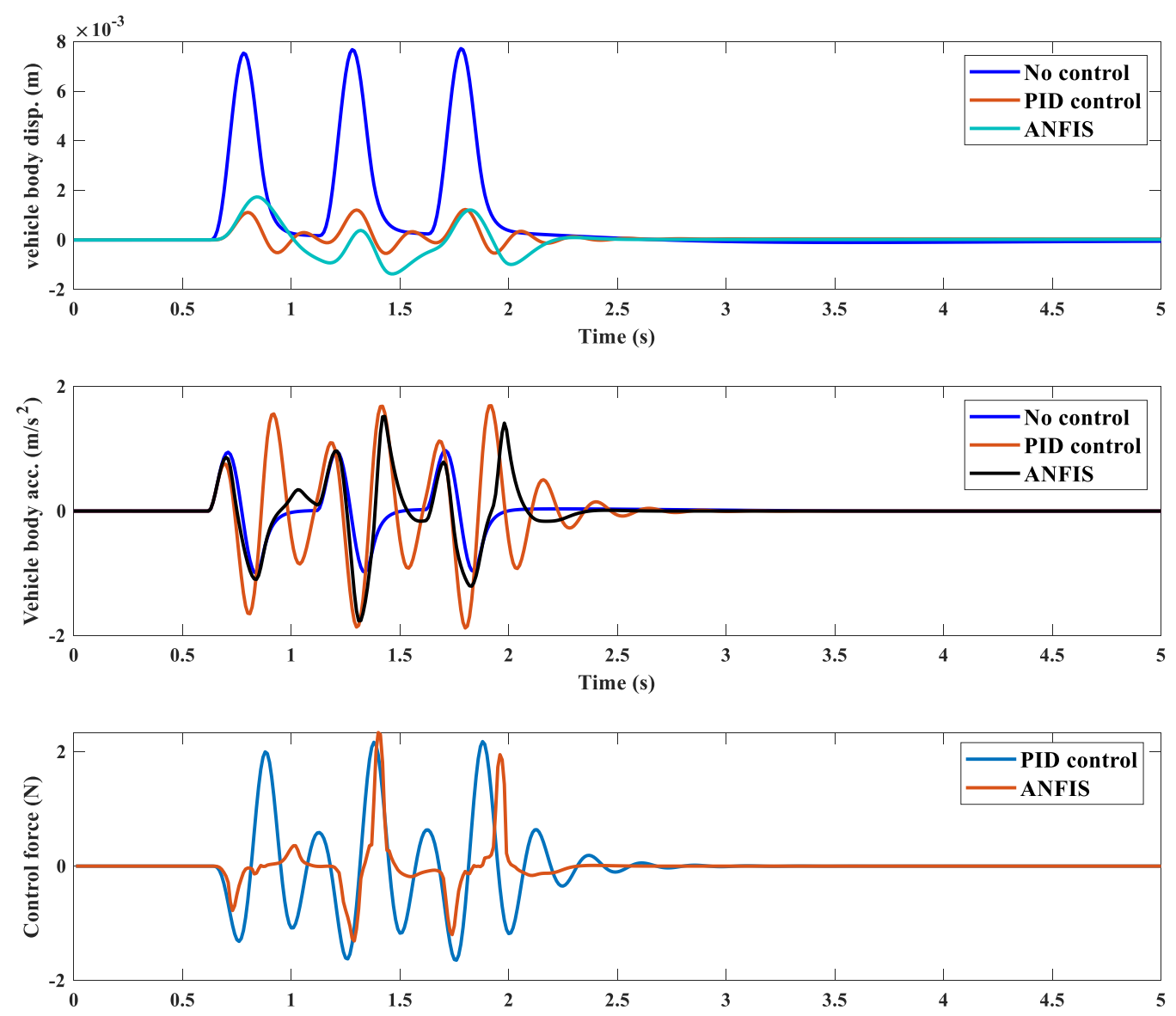

Figure 5. The vehicle dynamics comparison for the PID and ANFIS control algorithm.

\section{Conclusions}

The 3-DOFs quarter car vehicle model with the vehicle tire, vehicle suspension, and the vehicle body is studied. The vehicle considered moving on the rigid road in which three bumps included to surface. For the controlling vehicle body vibration, the PID and ANFIS control algorithm has been designed. The ANFIS training and testing data have been obtained from the PID control algorithm. Then, the performance of the ANFIS is compared with the PID algorithm. Consequently, it is proven that using the PID data for the ANFIS control algorithm is very effective in terms of the 3-DOFs quarter vehicle vibrations effect of rigid road with the nonrandom bumps.

\section{References}

[1] Koç MA, Esen İ. Modelling and analysis of vehicle-structure-road coupled interaction considering structural flexibility, vehicle parameters and road roughness. J Mech Sci Technol 2017;31. https://doi.org/10.1007/s12206-017-0403-y.

[2] Pacejka HB. Tire Characteristics and Vehicle Handling and Stability. 2012. 
https://doi.org/10.1016/b978-0-08-097016-5.00001-2.

[3] Ventsel E, Krauthammer T. Thin plates and shells : theory, analysis, and applications. Marcel Dekker; 2001.

[4] Kokowski P, Makarewicz R. Predicted effects of a speed bump on light vehicle noise. Appl Acoust 2006;67:570-9. https://doi.org/10.1016/j.apacoust.2005.10.001.

[5] Salau TAO, Adeyefa AO, Oke SA. Vehicle speed control using road bumps. Transport 2004;19:130-6. https://doi.org/10.1080/16484142.2004.9637965.

[6] Yang S-C, Jang S-Y. Investigation of the Maintenance Criteria for the Rail Surface Defects in High-Speed Railways. J Korean Soc Railw 2011;14:535-44. https://doi.org/10.7782/jksr.2011.14.6.535.

[7] Zhu M, Cheng X, Miao L, Sun X, Wang S. Advanced Stochastic Modeling of Railway Track Irregularities. Adv Mech Eng 2013;2013. https://doi.org/10.1155/2013/401637.

[8] Valášek M, Kortüm W, Šika Z, Magdolen L, Vaculín O. Development of semi-active road-friendly truck suspensions. Control Eng Pract 1998;6:735-44. https://doi.org/10.1016/S0967-0661(98)00079-3.

[9] Weiss EJ. The New Securities Fraud Pleading Requirement: Speed Bump or Road Block?, 1998.

[10] Pau M, Angius S. Do speed bumps really decrease traffic speed? An Italian experience. Accid Anal Prev 2001;33:585-97. https://doi.org/https://doi.org/10.1016/S00014575(00)00070-1.

[11] Elvik R. Area-wide urban traffic calming schemes: A meta-analysis of safety effects. Accid Anal Prev 2001;33:327-36. https://doi.org/10.1016/S0001-4575(00)00046-4.

[12] Zhu P, Hessling P, Liu D. Optimal road hump for comfortable speed reduction. Proc SPIE - Int Soc Opt Eng 2008;7130. https://doi.org/10.1117/12.819725.

[13] Guenounou O, Dahhou B, Athmani B. Optimal design of PID controller by Multiobjective genetic algorithms. n.d.

[14] Carlucho I, De Paula M, Acosta GG. Double Q-PID algorithm for mobile robot control. Expert Syst Appl 2019;137:292-307. https://doi.org/10.1016/J.ESWA.2019.06.066.

[15] Baxter R, Hastings N, Law a., Glass EJ. [ No Title ]. Anim Genet 2008;39:561-3. 